\title{
Radiative Mixed Convection Mass Transfer Flow past an ISO-thermal Porous Plate Embedded in a Permeable Medium in Presence of Thermal Diffusion and Heat Generation
}

\author{
Sanjib Sengupta \\ Department of Mathematics \\ Assam University, Silchar \\ Assam, India
}

\begin{abstract}
In this paper an exact analysis to study the combined effects of thermal diffusion (Soret) and heat generation on a mixed convection flow past an infinite vertical porous plate in presence of thermal radiation has been made. The governing system of partial differential equations subject to favorable boundary conditions is being solved analytically. CoglyVincentine-Gilles equilibrium approximations have been used to describe the radiative heat flux in the energy equation. Closed form of solutions for the velocity field, the temperature field and the concentration field are obtained and discussed graphically for various values of the physical parameters such as radiation parameter, Soret number, Grashof number, modified Grashof number, Prandtl number, Schmidt number and heat generation parameter. Moreover, expressions for the skin-friction, heat transfer co-efficient and mass transfer co-efficient are discussed with graphs and tables.
\end{abstract}

\section{Keywords}

Mixed convection, Mass transfer, Heat Source, Thermal diffusion, Thermal radiation.

\section{INTRODUCTION}

The process of heat transfer caused by simultaneous effect of free (natural) and forced convection is known as mixed convection flow. In industries and chemical engineering processes, there arise several situations, where a system contains two or more components whose concentrations vary from point to point and in such a system there is a natural tendency for mass to be transferred thereby minimizing the concentration differences within the system. The phenomenon, where one constituent from higher concentration region transport to a lower concentration region, is termed as mass transfer. Several authors have studied the case of mixed convection mass transfer viscous fluid flow of them Ostrach [1], Cheng [2], Bakier [3], Alam and Rahman [4], Chamkha and Ben-Nakhi [5] are notable contributors. It has observed that a mass flux can be generated by temperature gradient also, known as Soret effect or thermal-diffusion effect. Researchers like Durusunkaya and Worek [6], Kafousias and Williams [7],Anghel et al.[8] ,Alam and Rahman [9], Alam et al.[10], Ahmed and Sengupta [11] have studied Soret effect on hydromagnetic flow. Very recently Sengupta [12], has investigated the Soret effect on free convection mass transfer flow past a uniformly accelerated porous plate in presence of heat absorption. In all of these studies, the effect of heat generation and thermal radiation in the energy equation got little attention by the researchers. It has been seen that, the study of heat generation in moving fluids is important as it changes the temperature distribution and the particle deposition rate particularly in nuclear reactor cores, fire and combustion modeling, electronic chips and semi conductor wafers. It is also found that at high operating temperature, radiation effect can be quite significant and can't be ignored. The knowledge of thermal radiation plays a vital role particularly in nuclear power plants, gas turbines and the various propulsion devices for aircraft, missiles, satellites and space vehicles. Notable contribution in this regard was put forward by researchers, like Soundalgekar and Takhar [13], Hossain and Takhar [14], Raptis and Perdikis [15], El-Arabawy [16].

The objective of the discussion is to study the combined effects of the thermal-diffusion (Soret), heat generation and thermal radiation on a two-dimensional mixed convection viscous incompressible fluid past an infinite porous plate embedded in a permeable medium with constant suction.

\section{MATHEMATICAL FORMULATION AND SOLUTION OF THE PROBLEM}

A case of steady laminar flow of a viscous incompressible fluid past an infinite vertical porous flat plate is considered for discussion. Introducing a co-ordinate system $(\bar{x}, \bar{y})$ with $\bar{x}$-axis along the length of the plate in the upward vertical direction and $\bar{y}$-axis normal to the plate towards the fluid region. The plate is supposed to be non-reacting and subjecte to a constant suction parallel to $\bar{y}$-axis. The viscous dissipations of energy are assumed to be negligible for the study. Since, the plate is infinite in length all the fluid property except possibly the pressure remain constant along the $\bar{x}$-direction. Under Boussinesq approximations, the fluid property variation with temperature is limited only to density variation and the influence of variation of density with temperature is restricted to the body force term only. Considering the Boussinesq approximations and the usual boundary layer approximations, the equations governing the steady laminar two-dimensional mixed convection flow with medium concentration in presence of Soret and heat genaration effects reduce to: 


\section{Continuity Equation}

$$
\frac{\partial \bar{v}}{\partial \bar{y}}=0
$$

\section{Momentum Equation}

$\bar{v} \frac{\partial \bar{u}}{\partial \bar{y}}=v \frac{\partial^{2} \bar{u}}{\partial \bar{y}^{2}}+g \beta\left(\bar{T}-\bar{T}_{\infty}\right)+g \beta^{*}\left(\bar{C}-\bar{C}_{\infty}\right)+\frac{v}{\bar{K}}(\bar{U}-\bar{u})_{(2)}$

\section{Energy Equation}

$\bar{v} \frac{\partial \bar{T}}{\partial \bar{y}}=\frac{k}{\rho c_{p}} \frac{\partial^{2} \bar{T}}{\partial \bar{y}^{2}}-\frac{1}{\rho c_{p}} \frac{\partial \bar{q}_{\bar{y}}}{\partial \bar{y}}+\frac{Q_{0}}{\rho c_{p}}\left(\bar{T}-\bar{T}_{\infty}\right)$

\section{Species Continuity Equation}

$\bar{v} \frac{\partial \bar{C}}{\partial \bar{y}}=D_{M} \frac{\partial^{2} \bar{C}}{\partial \bar{y}^{2}}+D_{T} \frac{\partial^{2} \bar{T}}{\partial \bar{y}^{2}}$

The relevant boundary conditions are:

$$
\begin{aligned}
& \bar{y}=0: \overline{\mathrm{u}}=0, \overline{\mathrm{v}}=-V_{0}, \overline{\mathrm{T}}=\overline{\mathrm{T}}_{w}, \overline{\mathrm{C}}=\overline{\mathrm{C}}_{w} \\
& \bar{y} \rightarrow \infty: \overline{\mathrm{u}} \rightarrow \bar{U}_{0}, \overline{\mathrm{v}} \rightarrow-V_{0}, \overline{\mathrm{T}} \rightarrow \overline{\mathrm{T}}_{\infty}, \overline{\mathrm{C}} \rightarrow \overline{\mathrm{C}}_{\infty}
\end{aligned}
$$

The radiaive heat flux is quantified due to Cogly-Vincentine Gilles [17] differential form for non-gray gas near equilibrium as:

$$
\frac{\partial \bar{q}_{r \bar{y}}}{\partial \bar{y}}=4 I^{*}\left(\bar{T}-\bar{T}_{\infty}\right)
$$

Where, $\quad I^{*}=\int_{0}^{\infty} K_{y w}\left(\frac{\partial e_{b y}}{\partial \bar{T}}\right)_{w} d y$

Using (6), (3) gives

$\bar{v} \frac{\partial \bar{T}}{\partial \bar{y}}=\frac{k}{\rho c_{p}} \frac{\partial^{2} \bar{T}}{\partial \bar{y}^{2}}-\frac{4 I^{*}}{\rho c_{p}}\left(\bar{T}-\bar{T}_{\infty}\right)+\frac{Q_{0}}{\rho c_{p}}\left(\bar{T}-\bar{T}_{\infty}\right)$

We introduce the following non-dimensional quantities as:

$$
\begin{gathered}
y=\frac{V_{0} \bar{y}}{v}, \quad u=\frac{\bar{u}}{V_{0}}, \quad U=\frac{\bar{U}}{\bar{V}_{0}} \quad v=\frac{\bar{v}}{V_{0}} \quad \theta=\frac{\bar{T}-\bar{T}_{\infty}}{\bar{T}_{w}-\bar{T}_{\infty}} \\
\phi=\frac{\bar{C}-\bar{C}_{\infty}}{\bar{C}_{w}-\bar{C}_{\infty}}, S r=\frac{D_{T}\left(\bar{T}_{w}-\bar{T}_{\infty}\right)}{v\left(\bar{C}_{w}-\bar{C}_{\infty}\right)}, \operatorname{Pr}=\frac{v \rho c_{p}}{k}, \lambda=\frac{4 I^{*} v^{2}}{k V_{0}^{2}}
\end{gathered}
$$$$
G_{r}=\frac{g \beta v\left(\bar{T}_{w}-\bar{T}_{\infty}\right)}{V_{0}^{3}} G_{m}=\frac{g \beta^{*} v\left(\bar{C}_{w w}-\bar{C}_{\varphi}\right)}{V_{0}^{3}} S c=\frac{v}{D_{M}}
$$

$K=\frac{\bar{K} V_{0}^{2}}{v^{2}}, \quad S=\frac{Q_{0} v^{2}}{k V_{0}^{2}}\left(\varrho_{0}\right)$

The corresponding non-dimensional form of equations are:

$\frac{d v}{d y}=\mathrm{O}$

$\frac{d^{2} u}{d y^{2}}+\frac{d u}{d y}-\frac{1}{K} u=-G_{r} \theta-G_{m} \phi-\frac{U}{K}$

$\frac{d^{2} \theta}{d y^{2}}+\operatorname{Pr} \frac{d \theta}{d y}-\theta(S+\lambda)=0$

$\frac{d^{2} \phi}{d y^{2}}+S c \frac{d \phi}{d y}+S c S r \frac{d^{2} \theta}{d y^{2}}=0$

The non-dimensional boundary conditions are:

$$
\begin{aligned}
& \mathrm{y}=0: \mathrm{u}=0, \quad \mathrm{v}=-1, \quad \theta=1, \phi=1 \\
& \mathrm{y} \rightarrow \infty: \mathrm{u} \rightarrow \mathrm{U}, \mathrm{v} \rightarrow-1, \theta \rightarrow 0, \phi \rightarrow 0 .
\end{aligned}
$$

The solutions of (9), (10), (11) subject to (12) are,

$$
\begin{aligned}
& \mathrm{u}(\mathrm{y})=A_{9} e^{-A_{1} y}+A_{10} e^{-S c y}+A_{11} e^{-A_{4} y}+U \\
& \theta(\mathrm{y})=e^{-A_{1} y} \\
& \phi(\mathrm{y})=A_{2} e^{-A_{1} y}+A_{3} e^{-S c y}
\end{aligned}
$$

Where,

$$
\begin{aligned}
& \left.A_{1}=\frac{\operatorname{Pr}+\sqrt{\operatorname{Pr}^{2}+4(S+\lambda)}}{2}, \quad A_{2}=\frac{S c S r A_{1}}{S c-A_{1}} \operatorname{Sc} \neq A_{1}\right) \\
& A_{3}=1-A_{2}, \quad A_{4}=\frac{1+\sqrt{1+\frac{4}{K}}}{2}
\end{aligned}
$$$$
A_{5}=\frac{G r}{A_{1}^{2}-A_{1}-\frac{1}{K}} \quad A_{6}=\frac{G m}{S c^{2}-S c-\frac{1}{K}}
$$$$
A_{7}=\frac{S c S r G m}{\left(A_{1}^{2}-A_{1}-\frac{1}{K}\right)\left(S c-A_{1}\right)}
$$$$
A_{8}=\frac{S c S r G m}{\left(S c^{2}-S c-\frac{1}{K}\right)\left(S c-A_{1}\right)}, \quad\left(\mathrm{Sc} \neq A_{1}\right)
$$ 


\section{Non-dimensional rate of heat transfer}

$\mathrm{Nu}=-\frac{1}{\operatorname{Pr}}\left(\frac{\partial \theta}{\partial y}\right)_{y=0}=\frac{A_{1}}{\operatorname{Pr}}$

\section{Non-dimensional rate of mass transfer}

$$
\mathrm{Sh}=-\frac{1}{S c}\left(\frac{\partial \phi}{\partial y}\right)_{y=0}=\frac{1}{S c}\left(A_{13}+A_{3} S c\right)
$$

\section{RESULTS AND DISCUSSIONS:}

During the course of discussion of the effects of various physical parameters on the flow field, the following considerations are made:

(1) The value of the Prandtl number Pr is taken as 0.71 , which corresponds physically to air.

(2) The value of the Schmidt number Sc is chosen at 0.66, which represents water vapour at $25 \mathrm{C}$ and $1 \mathrm{~atm}$.

(3) The values of the Grashof number Gr , modified Grashof number $\mathrm{Gm}$, permeability parameter $\mathrm{K}$, non-dimensional free stream velocity $U$ are taken to be fixed at $5,5,1,1$ respectively.

(4) Finally the values of the Soret number $\mathrm{Sr}$, heat generation parameter $S$, radiation parameter $\lambda$ are chosen arbitrarily.

Under the above considerations, results are shown in figures 1-4 and in tables 1-3. Figure 1 shows the parametric effect of the thermal radiation parameter $\lambda$ on the non-dimensional temperature $\theta$. Due to the presence of the radiation parameter, the thickness of the thermal boundary layer decreases there by reducing the value of $\theta$. The change in the values of nondimensional concentration $\varphi$, due to change in values of radiation parameter $\lambda$ are shown in figure2. Though the effect of thermal radiation on concentration is found to be negligible, but it has observed that, $\varphi$ rises initially due to increase in values of $\lambda$ up to $0<y \leq 1.5$, thereafter declining narrowly when $y>1.5$. In figure 3 , the effects of Soret number $\mathrm{Sr}$ on the flow velocity $\mathrm{u}$ are shown. It is seen that, due to increase in values of $\mathrm{Sr}$, the fluid velocity u increases sharply near the plate and after that decreases steadily far away from the plate. This is in consistent with the fact that, an increase in the parameter $\mathrm{Sr}$ produces an increase in the mass buoyancy force, which results in increasing the value of $u$. Figure 4 depicts the graphical representation of the variation of the velocity $u$ due to change in values of thermal radiation parameter $\lambda$. The presence of radiation parameter helps in decreasing the thermal buoyancy force, which results in reducing the rate of flow and thus the fluid velocity. The numerical values of non-dimensional skin-friction $\tau$ for different values of radiation parameter $\lambda$ against arbitrary values of soret number $\mathrm{Sr}$ has been demonstrated in table 1 . The wall friction is found to be decreased by the presence of radiation parameter $\lambda$, where as reverse phenomena has observed due to Soret parameter Sr. Table 2 demonstrate numerically the effects of radiation parameter $\lambda$ on the nondimensional rate of heat transfer coefficient $\mathrm{Nu}$ against arbitrary values of heat generation parameter S. It clearly shows that, the presence of the parametrs $\lambda$ and $S$ encourages the rate of heat flow from plate to the fluid region. Table 3 depicts how the rate of mass transfer Sh being effected by the influence of radiation parameter $\lambda$ and the heat generation parameter S. It has found that Sh declyning narrowly due to radiation parameter $\lambda$ in a region where $\lambda \in(0,1]$ and thereafter falls gradually when $\lambda>1$, making Sh $<0$, thereby changes the direction of mass flow.

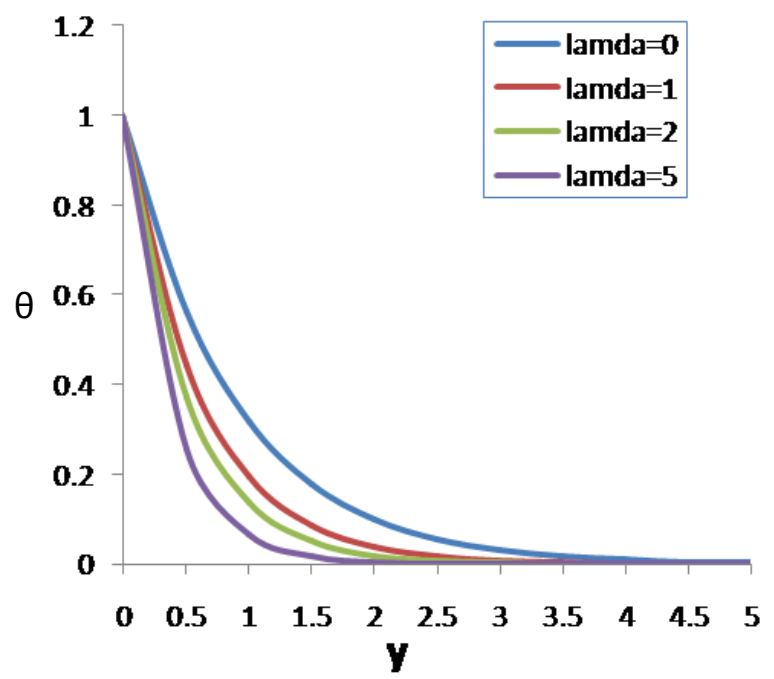

Figure1: Temperature $\theta$ versus y for $\mathrm{Pr}=0.71, \mathrm{~S}=0.5, \mathrm{Sc}=$ $0.22, G_{r}=4.0, G_{m}=5.0, U=1.0, S r=0.5, K=0.5$.

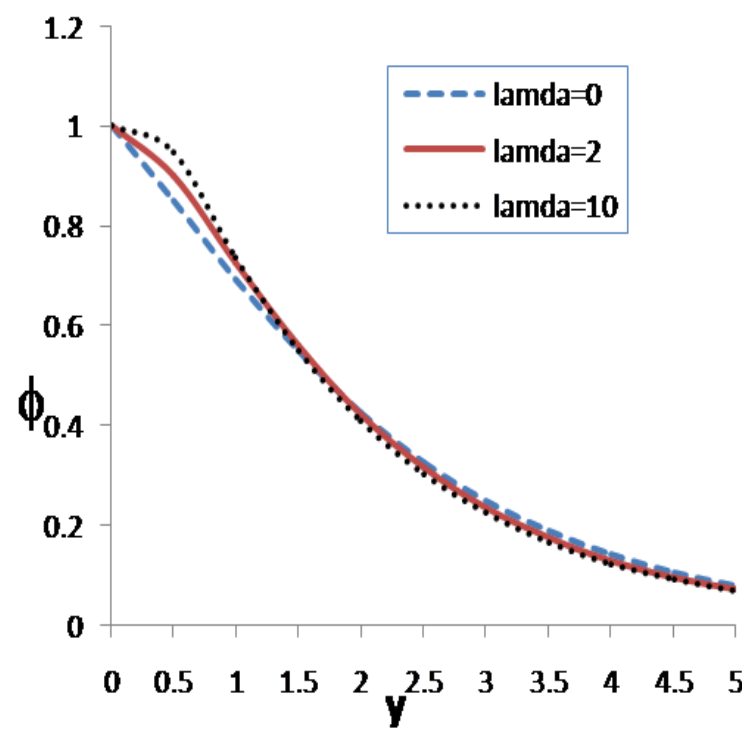

Figure 2: Concentration $\phi$ versus y for $\operatorname{Pr}=0.71$, $S c=0.22, G_{r}=4.0, G_{m}=1.0, U=1.0, S=1.0, S r=.5, K=0.5$ 


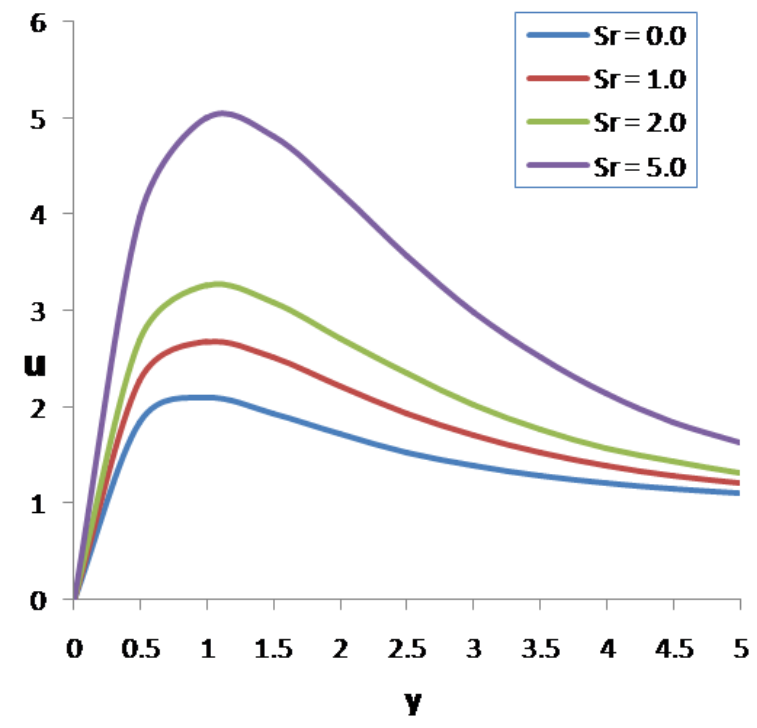

Figure 3: Velocity $u$ versus $y$ for $\operatorname{Pr}=0.71, S c=0.6, G_{r}$ $=5.0, G_{m}=5.0, U=1.0, S=1.0, K=0.5, \lambda=1.0$.

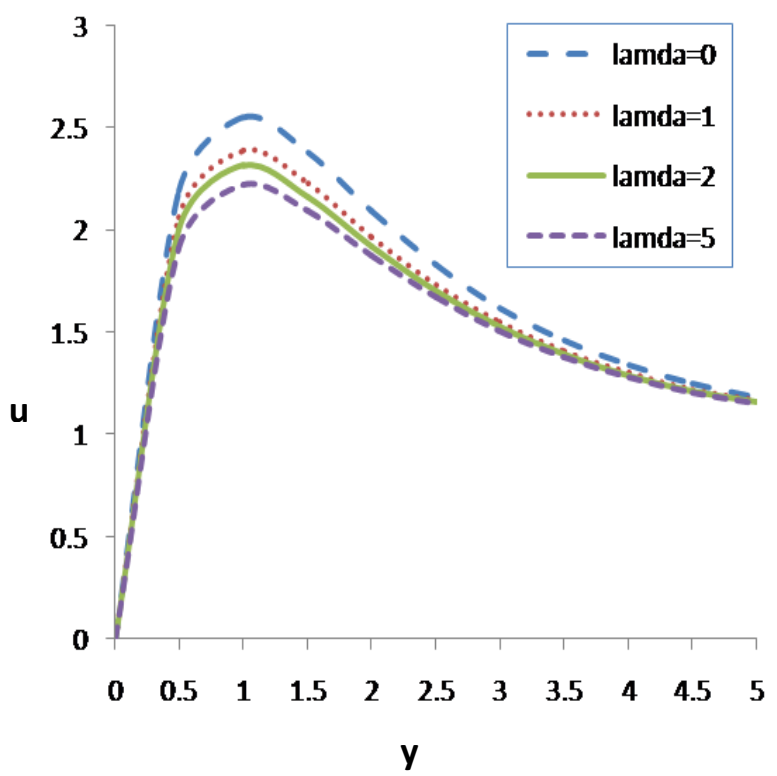

Figure 4: Velocity u versus y for $\operatorname{Pr}=0.71, S c=0.6, G_{r}$ $=5.0, G_{m}=5.0, U=1.0, S=1.0, K=0.5, S r=0.5$.
Table1.Numerical values of non-dimensional skin-friction $\tau$ for different values of radiation parameter $\lambda$ against arbitrary values of Soret number $\mathrm{Sr}$ and for fixed values of $\operatorname{Pr}=0.71, G_{r}=5.0, G_{m}=5.0, U=1.0 S=.5, K=.5, S c=.6$, $\mathbf{y}=\mathbf{0}$.

\begin{tabular}{|l|l|l|l|}
\hline Sr & $\lambda=\mathbf{0}$ & $\boldsymbol{\lambda}=\mathbf{1}$ & $\boldsymbol{\lambda}=\mathbf{5}$ \\
\hline 0.0 & 7.455 & 7.026 & 6.467 \\
\hline 0.5 & 7.955 & 7.607 & 7.153 \\
\hline 1.0 & 8.456 & 8.188 & 7.838 \\
\hline 1.5 & 8.957 & 8.769 & 8.524 \\
\hline 2.0 & 9.457 & 9.350 & 9.210 \\
\hline 2.5 & 9.958 & 9.931 & 9.896 \\
\hline 3.0 & 10.459 & 10.512 & 10.582 \\
\hline 3.5 & 10.959 & 11.093 & 11.268 \\
\hline 4.0 & 11.460 & 11.674 & 11.954 \\
\hline 4.5 & 11.961 & 12.256 & 12.640 \\
\hline 5.0 & 12.462 & 12.837 & 13.326 \\
\hline & & & \\
\hline
\end{tabular}

Table 2.Numerical values of non-dimensional rate of heat transfer coefficient $\mathrm{Nu}$ for different values of radiation parameter $\lambda$ against arbitrary values of heat generation parameter $S$ and for fixed values of $\operatorname{Pr}=0.71, G_{r}=5.0$, $\mathrm{G}_{\mathrm{m}}=5.0, \mathrm{U}=1.0, \mathrm{Sc}=.6, \mathrm{~K}=.5, \mathrm{Sr}=.5, \mathrm{y}=0$.

\begin{tabular}{|l|l|l|l|}
\hline Sr & $\lambda=\mathbf{0}$ & $\lambda=\mathbf{1}$ & $\lambda=\mathbf{5}$ \\
\hline 0.0 & 1.995 & 2.554 & 3.689 \\
\hline 0.1 & 2.060 & 2.601 & 3.720 \\
\hline 0.2 & 2.122 & 2.648 & 3.750 \\
\hline 0.3 & 2.182 & 2.694 & 3.780 \\
\hline 0.4 & 2.240 & 2.739 & 3.811 \\
\hline 0.5 & 2.296 & 2.782 & 3.841 \\
\hline 0.6 & 2.350 & 2.825 & 3.870 \\
\hline 0.7 & 2.403 & 2.868 & 3.900 \\
\hline 0.8 & 2.455 & 2.909 & 3.929 \\
\hline 0.9 & 2.505 & 2.950 & 3.957 \\
\hline 1.0 & 2.554 & 2.990 & 3.986 \\
\hline & & & \\
\hline
\end{tabular}


Table 3. Demonstration of numerical values of rate of mass transfer Sh for different values of radiation parameter $\lambda$ against arbitrary values of heat generation parameter $S$ and for fixed values of $\operatorname{Pr}=.71 G_{r}=5.0$, $\mathrm{G}_{\mathrm{m}}=5.0, \mathrm{U}=1.0 \mathrm{Sr}=.5, \mathrm{~K}=.5, \mathrm{Sc}=.6, \mathrm{y}=0$.

\begin{tabular}{|l|l|l|l|}
\hline $\mathbf{S r}$ & $\lambda=\mathbf{0}$ & $\lambda=\mathbf{1}$ & $\lambda=\mathbf{5}$ \\
\hline 0.0 & 0.292 & 0.093 & -0.310 \\
\hline 0.1 & 0.269 & 0.077 & -0.321 \\
\hline 0.2 & 0.247 & 0.060 & -0.331 \\
\hline 0.3 & 0.225 & 0.044 & -0.342 \\
\hline 0.4 & 0.205 & 0.028 & -0.353 \\
\hline 0.5 & 0.185 & 0.012 & -0.363 \\
\hline 0.6 & 0.166 & -0.003 & -0.374 \\
\hline 0.7 & 0.147 & -0.018 & -0.384 \\
\hline 0.8 & 0.129 & -0.033 & -0.395 \\
\hline 0.9 & 0.111 & -0.047 & -0.405 \\
\hline 1.0 & 0.093 & -0.062 & -0.415 \\
\hline
\end{tabular}

\section{CONCLUSIONS}

A theoretical study is performed to discuss the influence of thermal radiation on a incompressible viscous fluid past a vertical porous plate in presence of Soret and heat genaration effects. The investigation leads to the following conclusions:

[1] The flow is accelerated under the influence of Soret effect and retarded due to thermal radiation.

[2] The temperature falls due to radiation effect .

[3] The concentration first increases and thereafter decreases due to the presence of thermal radiation.

[4] The skin-friction decreases in presence of radiation effect and increases due to Soret effect.

[5] The rate of heat transfer raises due to the influence of radiation and heat genaration effects.

[6] The mass transfer rate declyning gradually and becomes negative thereby changing the direction of mass transfer in presence of thermal radiation and heat genaration .

\section{LIST OF SYMBOLS}

$\bar{C}$ Species concentration

$C_{p}$ Specific heat at constant pressure

$\bar{C}_{w}$ Species concentration at the plate

$\bar{C}_{\infty}$ Species concentration in the free stream

$D_{M}$ Co-efficient of mass diffusion

$D_{T} \quad$ Co-efficient of thermal diffusion

g Acceleration due to gravity
$G_{m} \quad$ Grashof number for mass transfer

$G_{r} \quad$ Grashof number for heat transfer

k Thermal conductivity

K Permeability parameter

$\mathrm{Nu}$ Nusselt number

Pr Prandtl number

$\bar{q}_{r \bar{y}}$ Radiative component of heat flux along

$\bar{y}$ direction

$Q_{0}$ Heat source parameter (dimensional)

$\mathrm{S}$ Heat source parameter(non-dimensional)

Sc Schmidt number

Sh Non-dimensional Sherwood number

$\mathrm{Sr}$ Soret number

$\bar{T} \quad$ Fluid temperature

$\bar{T}_{w} \quad$ Temperature at the plate

$\bar{T}_{\infty} \quad$ Temperature in the free stream

$\bar{U} \quad$ Free Stream velocity (dimensional)

U Free Stream velocity (non-dimensional)

$V_{0} \quad$ Suction velocity

$(\bar{u}, \bar{v})$ Velocity components (dimensional)

$(\mathrm{u}, \mathrm{v})$ Velocity components (nondimensional)

$(\bar{x}, \bar{y})$ Cartesian Co-ordinate (dimensional)

$(\mathrm{x}, \mathrm{y})$ Cartesian Co-ordinate (nondimensional)

\section{Greek Symbols}

$\beta \quad$ Co-efficient of volume expansion for thermal expansion

$\beta^{*}$ Co-efficient of volume expansion for mass expansion

$\rho \quad$ Density of the fluid

$\theta \quad$ Non dimensional temperature

$v \quad$ Kinematic Co-efficient of viscosity

$\phi \quad$ Non dimensional Species Concentration

$\tau \quad$ Non- dimensional skin friction

$\lambda \quad$ Radiative heat flux constant

\section{ACKNOWLEDGMENT}

The author thanks the referee for his valuable comments.

\section{REFERENCES}

[1] Ostrach, S. 1954.Combined natural and forced convection laminar flow and heat transfer of fluids with and without heat sources in channels with linearly varying wall temperatures. NACA TN 3141.

[2] Cheng, P. 1977.Combined free and forced boundary layer flows about inclined surfacesin a porous medium, International Journal of Heat and Mass Transfer 20, 806814. 
[3] Bakier, A.Y. 2001.Thermal radiation effect on mixed convection from vertical surfaces in saturated porous media. International Communications in Heat and Mass Transfer 28, 119-126.

[4] Alam, M.S., Rahman,.M.M. 2006.Dufour and Soret effects on mixed convection flow past a vertical porous flat plate with variable suction. Nonlinear Analysis: Modelling and Control, 11, 3-12.

[5] Chamkha, A.J., Ben-Nakhi, A.,. 2008.MHD mixed convection-radiation interaction along a permeable surface immersed in a porous medium in the presence of Soret and Dufour effects. International J. Of heat and mass transfer,44, 845-856.

[6] Dursunkaya,Z.,Worek,M.1992. Diffusion - thermo and Thermo-Diffusion effects in transient and steady natural convection from vertical surface. International Journal of heat and mass transfer, 35, 2060-2065.

[7] Kafoussias, N.G., Willams ,E.M. 1995. ThermalDiffusion and Diffusion-thermo effects on mixed freeforced convective and mass transfer boundary layer flow with temperature dependent viscosity. International Journal of Engineering Science, 33, 1369-1384.

[8] Anghel, M., Takhar, H.S., Pop, I. 2000.Dufour and Soret effects on free convection boundary layer flow over a vertical surface embedded in a porous medium. Studia universities, Babes Bolyai Mathematica, XLV, 11-21.

[9] Alam, M.S., Rahamann, M.M. 2005. Dufour and Soret effects on MHD free convection heat and mass transfer flow past a vertical flat plate embedded in a porous medium. Journal of Naval Architecture and Marine Engineering. 1, 55-65.

[10] Alam , S., Rahamann ,M .M., Maleque, A., Ferdows, M. 2006. Dufour and Soret effects on steady MHD combined free-forced convective and mass transfer flow past a semi-infinite vertical plate. Thammasat International Journal of Science and technology, 11, 112.

[11] Ahmed, N., Sengupta,S. 2011. Thermo-diffusion and diffusion-thermo effects on a three -dimensional mhd mixed convection flow past an infinite vertical porous plate with thermal radiation. J. of Magnetohydrodynamics, 47, 41-60.

[12] Sengupta, S. 2011. Thermal diffusion effect of free convection mass transfer flow past a uniformly accelerated porous plate with heat sink. International Journal of Mathematical Archive, 2, 1266-1273.

[13] Soundalgekar,V.M., Takhar,H.S. 1993. Radiation effects on free convection flow past a semi-infinite vertical plate. Modeling, measurement and control, B51, 31-40.

[14] Hossain M.A., Takhar H.S., 1996. Radiation effects on mixed convection along a vertical plate with uniform surface temperature. International Journal of heat and mass transfer, 31, 243-248.

[15] Raptis,A.,Perdikis,C.1999.Radiation and free convection flow past a moving plate.International Journal of heat and mass transfer, 4, 817-821.

[16] El-Arabawy, H.A.M. 2003. Effect of suction/injection on the flow of a micro polar fluid past a continuously moving plate in the presence of radiation, International Journal of heat and mass transfer, $46,1471-1477$.

[17] Cogly, A. C.,Vincentine, W.C, Gilles, SE. 1968. Differential approximation for radiative transfer in a non-gray gas near equilibrium. AIAA Journal, 6 , 551555 